\title{
Fluid-Structure Interaction Modelling of Left Ventricular Filling
}

\author{
Pascal R. Verdonck, Jan A. Vierendeels \\ Institute Biomedical Technology \\ Ghent University \\ Sint-Pietersnieuwstraat 41 \\ 9000 Ghent, Belgium
}

\begin{abstract}
Non-invasive diagnosis of diastolic dysfunction remains difficult in clinical practice. Non-invasive assessment of the flow field within the left ventricle (LV) using color M-mode Doppler (CMD) echocardiography provides a potential technique that can differentiate between the normal and diseased heart.

A computer model is developed describing three-dimensional axi-symmetrical LV filling flow. The simulation results show that the hydrodynamical mechanism of LV flow wave propagation, as observed on 2D color and color M-mode Doppler echocardiograms, is the propagation of a vortex in the LV cavity.
\end{abstract}

\section{Introduction}

Recently color M-mode Doppler echocardiography (CMD) has been proposed as a useful method for the evaluation of LV flow wave propagation as an index of LV filling [1-3]. The objective of this study is to obtain insight in the hydrodynamics of the color M-mode Doppler flow propagation velocity $\mathrm{v}(\mathrm{p})$ based on a mathematical framework. For the development of fluid-structure interaction model of the left ventricular filling the unsteady Navier-Stokes flow equations are solved in a LV truncated ellipsoid geometry with moving LV walls including relaxation and compliance of the wall. The computed results confirm both intraventricular flow and pressure patterns during filling. Vortices are formed during the acceleration phases of the early and atrial filling waves. During the deceleration phases the vortices are amplified and convected into the ventricle. The vortices are recognized on the derived 2D color echocardiograms as in vivo. The propagation of this vortex determines the propagation of the maximum velocity observed in the color M-mode Doppler echocardiogram.

Peskin [4] was the first to model the blood-ventricular wall interaction using the immersed boundary method. Owen [5] developed a 1D model showing the importance of intraventricular pressure wave propagation during early filling. In our previous work, a 1D model was constructed in order to simulate intraventricular pressure gradients in the LV during filling [6,7]. The present model, based on a 3D axisymmetrical computational approach [8], evaluates the fluid dynamics of the LV 
filling. The interaction between the LV wall and the blood is taken into account with a validated algorithm [8].

The calculated flow patterns in the LV are transformed into the format of $2 \mathrm{D}$ color and color M-mode Doppler echocardiograms as used in clinical practice. In this chapter we are studying in particular the relation between vortex center and position of maximum velocity along the centerline for both the early filling (E-wave) and atrial contraction wave (A-wave).

\section{Computer model}

\subsection{Intraventricular flow model}

The fluid domain of the LV is described by means of an axi-symmetrical model. The unsteady Navier-Stokes equations expressing conservation of momentum and the continuity equation expressing conservation of mass are solved in a LV truncated ellipsoid geometry with moving walls. The computation is started at the onset of LV relaxation. When the LV pressure drops below the atrial pressure, the mitral valve opens immediately. From this moment, a mitral velocity pattern is applied at the circular orifice (base) of the LV. It is assumed that forces of the mitral valve on the fluid are small and can be neglected once the mitral valve opens. After opening, pressure in the LV is determined by both the relaxation and compliance of the LV wall and the dynamics of the blood flow into the LV cavity. The computation ends before the LV contraction.

The mitral flow velocity pattern used as boundary condition and atrial pressure are derived from a separate calculation with Meisner's lumped parameter model [9].

\subsection{Left ventricular wall model}

The LV wall is described by a truncated ellipsoid in the zero stress state. At the zero stress state and with blood at rest, the transmural pressure is zero. The zero stress state is assumed to correspond with a cavity volume of $12 \mathrm{ml}$, diameter of mitral annulus of $1.5 \mathrm{~cm}$ and base to apex distance of $4 \mathrm{~cm}$. These are physiological relevant parameters for a canine heart for which the model was validated [8].

Away from the zero stress state, the shape of the LV is computed from equilibrium equations for the LV wall. These equilibrium equations involve the circumferential and longitudinal cardiac stresses, the curvature of the heart wall and the transmural pressure difference. A non-linear extension of the thin shell equations is used [8]. The position of the mitral valve annulus is kept fixed. 


\subsection{Boundary conditions}

We have used Meisner's model [9] at a heart rate of 80 beats per minute in order to obtain the necessary boundary conditions for our 3D model calculations: end systolic volume of $18 \mathrm{ml}$, end systolic pressure of $75 \mathrm{mmHg}$, time constant of relaxation $\tau_{\mathrm{v}}=30 \mathrm{~ms}$, left atrial pressure at opening of the mitral valve $6.75 \mathrm{mmHg}$ and the computed mitral velocity pattern as shown in figure 1.

\subsection{Coupling procedure}

The coupling of the heart wall displacement and the LV filling dynamics is based on an iterative approach [8]. For each time step $(1.5 \mathrm{~ms})$, the procedure alternates between the following steps: 1) calculation of the movement of the LV wall, for a known intraventricular pressure distribution along the wall, 2) calculation of the flow field inside the moving ventricle, which results in an updated velocity pattern and a new approximation of the pressure field to be used in step 1) until convergence for the time step is achieved.

\subsection{Computation}

Given the cardiac wall properties together with the circulation characteristics, the cardiac contraction and relaxation are the driving forces for the computation. First, the mitral inflow pattern, ESV and ESP and the left atrial pressure at opening of the mitral valve are computed with Meisner's model. Then, with the present model and with the same characteristics for the LV, the wall motion and the intraventricular pressure and flow patterns are computed during filling respecting the conservation of mass and momentum of the blood flow and the equilibrium equations for the heart wall. All details of the model can be found in [8].

\section{Results}

\subsection{Intraventricular pressure-time curves}

Figure 1 shows calculated intraventricular pressures in function of time (bottom panel) for the imposed mitral velocity pattern (top panel). Pressures curves at base (full line) and at a position inside the LV $4 \mathrm{~cm}$ from the base (dotted line) are compared. The pressure rise during early filling first occurs at the apex and then at the base. At the onset of atrial contraction, there is first a pressure rise at the base and then at the apex. 


\subsection{Simulated 2D color Doppler echocardiograms}

Figure 2 shows the corresponding flow velocity patterns and derived 2D color Doppler images at six different moments in time LV during filling. These moments are indicated with a black spot on the mitral velocity pattern: (1) before the immediate opening of the mitral valve, (2) at peak E velocity, (3) during deceleration of E-wave, (4) at diastasis, (5) at peak A velocity and (6) during deceleration of the A-wave. The flow pattern is shown and corresponds to the instantaneous velocity vectors in the LV cavity. The computed flow velocities are presented in a 2D color echo format for each of the six moments in the lower segment.

\subsection{Vortex propagation}

The filling is characterized by different vortex movements. A first vortex is formed before the maximum of the early filling wave occurs at the mitral annulus (figure 2, panel 2). The vortex is amplified during the deceleration phase of the early filling wave and moves into the LV towards the apex. Between E- and A-wave (diastasis) one vortex can be seen in the whole LV (panel 3). At the base a second vortex can be detected, with the direction of rotation opposite to the first vortex (panel 4). During the acceleration phase of the A-wave the second vortex grows, while a third vortex is formed, comparable to the first one (panel 5). During the deceleration phase of the Awave the second vortex dissappears. The first vortex is now located at the apex and the third one fills the basal region (panel 6). The vortices can be recognized in the 2D color echocardiograms by the presence of the transition of red to blue color near the cardiac wall, indicating backflow during filling.

In figure 3 (left panel), the positions of the vortex center and the positions of the maximum flow velocity along the centerline as a function of time are compared for both the E- and the A-wave. The right panel of figure 3 compares the position of the maximum flow velocity and the position of the vortex for both the E- and the A-wave.

\subsection{Simulated color M-mode Doppler Echocardiogram (CMD)}

Figure 4 shows a CMD along the symmetry axis, calculated by the present model corresponding to the velocities in figure 3. Both the early and atrial filling wave can be seen. During the initial flow phase blood moves almost simultaneously in the whole LV, which can alse be seen in the top part of figure 3, panel 2. This phase is denoted as I in figure 4 . The movement is caused by the passing of the pressure filling wave. The filling causes the generation of a vortex, as described earlier. The propagation of this vortex determines the propagation of the maximum velocity along the symmetry axis of the LV as can be seen in figures 3 and 4 (vortex propagation wave). The propagation of the maximum velocity or the propagation of the vortex is seen in the CMD and is denoted as phase II in figure 4. 


\section{Discussion}

A computer model is developed describing three-dimensional axisymmetrical LV filling flow. The calculated pressure-time curves in figure 1 confirm the existence of intraventricular pressure gradients. These gradients are also observed in dog studies [11] and in a 1D computer simulation of LV filling [6,7]. The minimal pressure in the apex is achieved shortly after the arrival of the pressure filling wave at the apex (denoted as I on the CMD in figure 4). At that moment the pressure rises at the apex since the pressure wave is reflected (reflection at a closed end) as already described by Owen [5].

Vortex formation during filling is an experimentally observed phenomenon [12-14] and according to Bellhouse [12] the presence of a vortex ring provides a mechanism for early valve closure in diastole. More recently, Steen and Steen [14] showed in vitro that at the start of filling, blood moves simultaneously at all positions, behaving as an incompressible fluid column (phase I). Then they observed a flow wave propagating from the mitral orifice towards the apex (phase II). In this phase, a ring vortex was seen to travel from the orifice towards the apex. Both phases are clearly observed in our simulation (figure 4). Shortland et al. [15] studied vortex formation and travelling in a skeletal muscle ventricle. They suggested that vortex travelling is an important feature to decrease the residence time of blood cells in the apical region. It is clear from figure 3 that the propagation of the maximum blood velocity inside the $\mathrm{LV}$, as it can be measured by CMD echocardiography, is associated to vortex propagation.

For the calculation shown in figure 4, the maximal blood velocity during the early filling wave is $70 \mathrm{~cm} / \mathrm{s}$ and $\mathrm{v}(\mathrm{p})$ of the filling wave, which corresponds with the propagation velocity of the ring vortex, is $45 \mathrm{~cm} / \mathrm{s}$. The ratio is 1.56 . This hemodynamic behaviour was also observed in vitro experiments [14] and in vivo measurements $[1-3,16]$.

\section{Conclusion}

A computer model is developed describing three-dimensional axisymmetrical LV filling flow. The simulation results show that the hydrodynamical mechanism of LV flow wave propagation, as observed on 2D color and color M-mode Doppler echocardiograms, is the propagation of a vortex in the LV cavity.

\section{Acknowledgements}

The research reported here was granted by contract GOA-95003 of the concerted action programme of the Ghent university, supported by the Flemish government. 


\section{References}

[1] Brun P, Tribouilloy C, Duval AM, Iseriu L, Meguira A, Pelle G, Dubois-Randé JL. Left ventricular flow propagation during early filling is related to wall relaxation: a color Mmode Doppler analysis. J. Am. Coll. Cardiol. 1992;20(2):420-32.

[2] Stugaard M, Risöe C, Halfdan I, Smiseth OA. Intracavitary filling pattern in the failing left ventricle assessed by color m-mode doppler echocardiography. J. Am. Coll. Cardiol. 1994;24(3):663-670.

[3] Takatsuji H, Mikami T, Urasawa K, Teranishi J-I, Onozuka H, Takagi C, Makita Y, Matsuo H, Kusuoka H, Kitabatake A. A new approach for evaluation of left ventricular diastolic function: Spatial and temporal analysis of ventricular filling flow propagation by color M-mode Doppler echocardiography. J. Am. Coll. Cardiol. 1996; 27(2):365-71.

[4] Peskin CS, McQueen DM. A three-dimensional computational method for blood flow in the heart I. immersed elastic fibers in a viscous incompressible fluid. J. Comp. Phys. 1989;81:372-405.

[5] Owen A. A numerical model of early diastolic filling: importance of intraventricular pressure wave propagation. Cardiovasc. Res. 1993;27:255-261.

[6] Vierendeels J, Verdonck P, Dick E. Intraventricular Pressure Gradients and the Role of Pressure Wave Propagation. J. Cardiovasc. Diagn. P. 1997;14(3):147-152.

[7] Verdonck PR, Vierendeels J, Riemslagh K, Dick E. Left Ventricular Pressure Gradients: a 1D Computer Model Simulation. Med. \& Biol. Eng. \& Comput. 1999;37(4):511-516.

[8] Vierendeels JA, Riemslagh K, Dick E, Verdonck PR. Computer Simulation of Intraventricular Flow and Pressure Gradients during Diastole, ASME J. Biomech. Eng. 2000;122(6):667-674.

[9] Meisner J. Left atrial role in left ventricular filling: dog and computer studies. Ph.D. thesis Albert Einstein College of Medicine. Yeshiva University, New York. 1986.

[10] Nishimura RA, Tajik AJ. Evaluation of diastolic filling of left ventricle in health and disease: Doppler Echocardiography is the clinician's Rosetta stone. J. Am. Coll. Cardiol. 1997;30:8-18.

[11] Courtois M, Kovács SJ Jr., Ludbrook PA. Transmitral pressure-flow velocity relation: importance of regional pressure gradients in the left ventricle during diastole. Circulation 1988;78:661-671.

[12] Bellhouse BJ. Fluid mechanics of a model mitral valve and left ventricle. Cardiovasc. Res. 1972;6:199-210.

[13] Lee CSF, Talbot L. A fluid-mechanical study of the closure of heart valves. J. Fluid. Mech. 1979;91(1):41-63.

[14] Steen T, Steen S. Filling of a model left ventricle studied by color M mode Doppler. Cardiovasc. Res. 1994;28:1821-1827.

[15] Shortland AP, Black RA, Jarvis JC, Henry FS, Iudicello F, Collins MW, Salmons S. Formation and travel of vortices in model ventricles: application to the design of skeletal muscle ventricles. J. Biomech. 1996;29:503-511.

[16] Greenberg NL, Vandervoort PM, Thomas JD. Instantaneous diastolic transmitral pressure difference from color Doppler M-mode echocardiography. Am. J. Physiol. 1996;271 (Heart Circ. Physiol. 40):H1267-H1276.

[17] Iudicello F, Henry FS, Collins MW, Salmons S, Sarti A, Lamberti C. Comparison of haemodynamic structures between a skeletal muscle ventricle and the human left ventricle, Internal Medicine, 1997;5:1-10.

[18] Nikolic S, Fenely M, Pajaro O, Rankin JS and Yellin E. Origin of regional pressure gradients in the left ventricle during early diastole. American Journal of Physiology, 1995;268:550-557. 


\section{Legends}

Figure 1. Top: Velocity profile at mitral valve. Bottom: Computed pressures at mitral valve and at a position inside the $\mathrm{LV} 4 \mathrm{~cm}$ from the mitral valve.

Figure 2. Flow patterns and calculated 2D color Doppler echocardiograms at six different time steps during filling: before opening of mitral valve, peak $\mathrm{E}$ velocity, deceleration of E-wave, diastasis, peak A velocity and deceleration of the A-wave

Figure 3. Relation between vortex center and position of maximum velolicty along the centerline for both E- and A-wave.

Figure 4. Color M-mode Doppler echocardiogram derived from a 2D axisymmetrical flow simulation. The scanline corresponds with the axis of symmetry. Both the early and atrial filling wave can be seen, the initial flow phase with blood moving simultaneously in the LV is denoted as I (pressure filling wave), the propagation of the vortex wave is denoted as II.
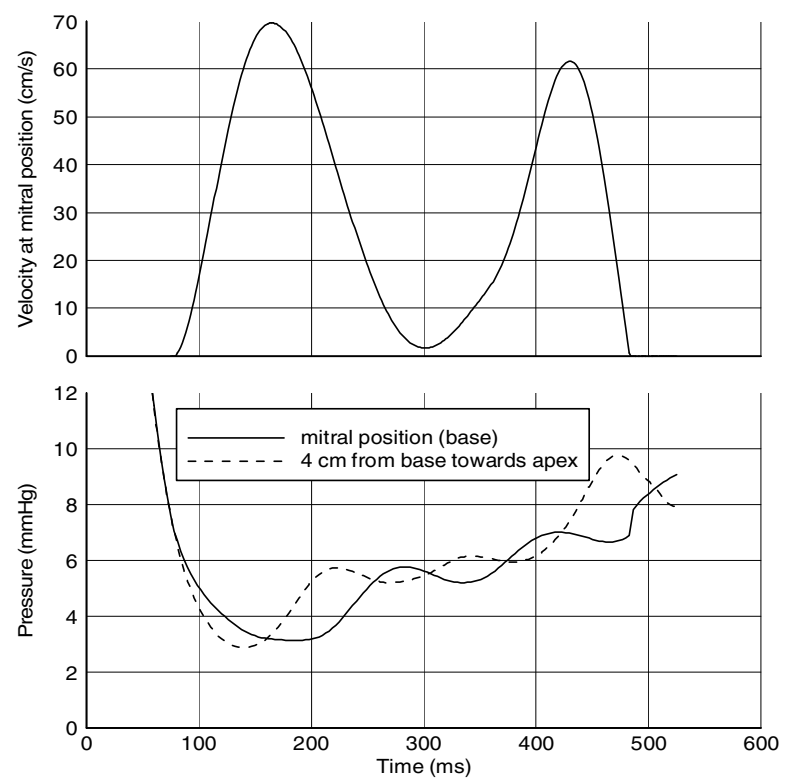

Fig. 1. Top: Velocity profile at mitral valve. Bottom: Computed pressures at mitral valve and at a position inside the $\mathrm{LV} 4 \mathrm{~cm}$ from the mitral valve. 


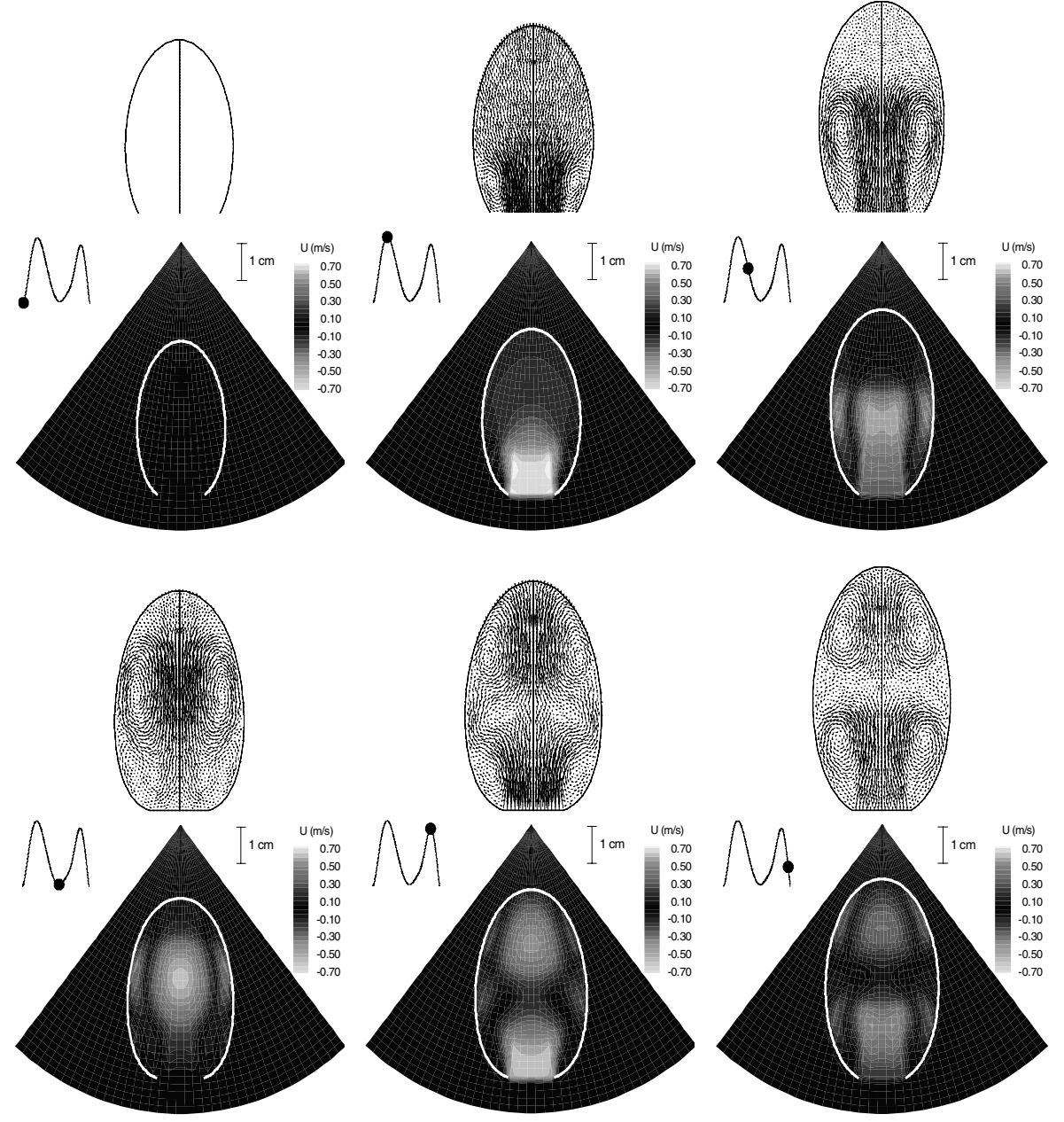

Fig. 2. Flow patterns and calculated $2 \mathrm{D}$ color Doppler echocardiograms at six different time steps during filling: before opening of mitral valve, peak $\mathrm{E}$ velocity, deceleration of E-wave, diastasis, peak A velocity and deceleration of the A-wave 

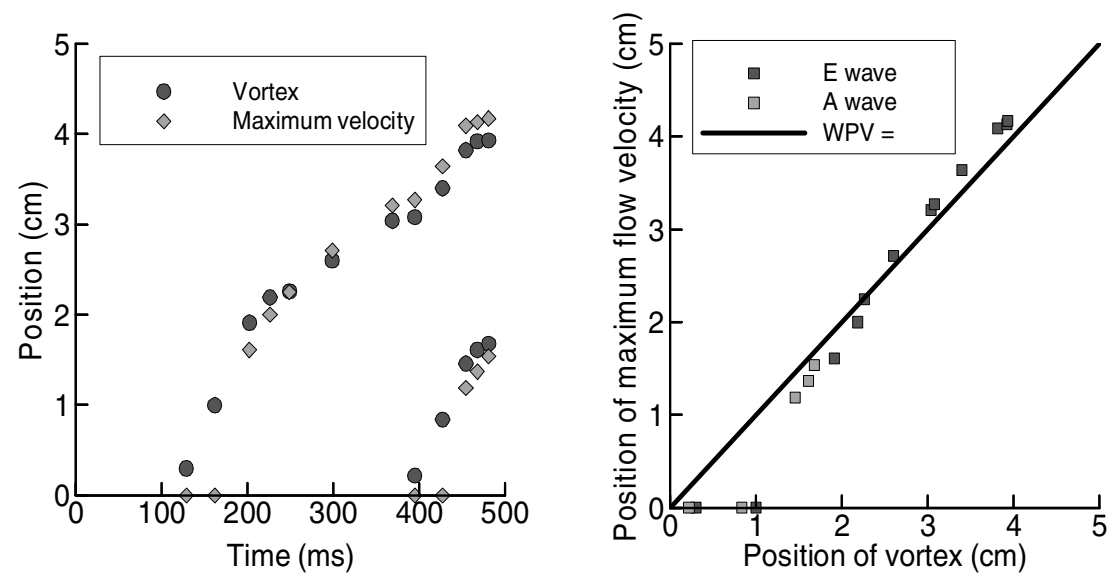

Fig. 3. Relation between vortex center and position of maximum velolicty along the centerline for both E- and A-wave. 


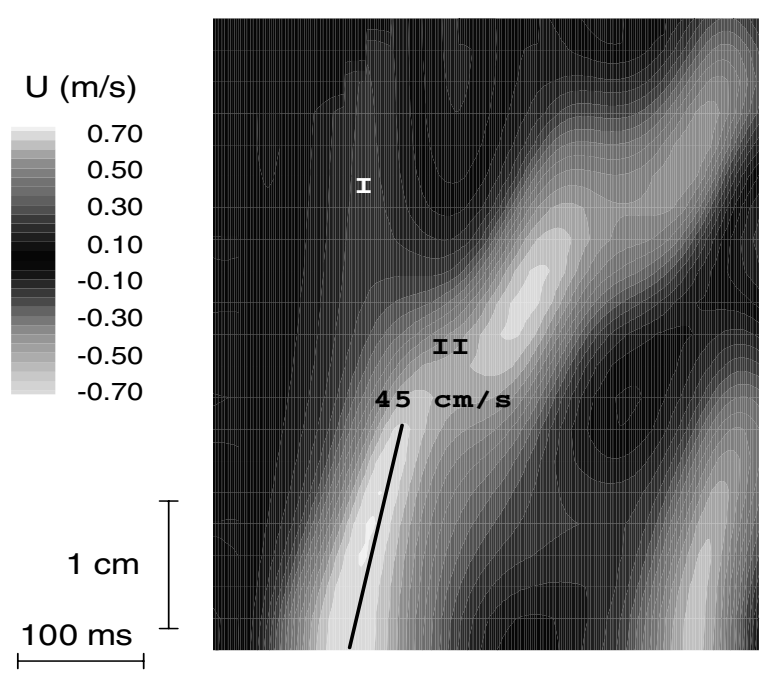

Fig 4. Color M-mode Doppler echocardiogram derived from a $2 \mathrm{D}$ axisymmetrical flow simulation. The scanline corresponds with the axis of symmetry. Both the early and atrial filling wave can be seen, the initial flow phase with blood moving simultaneously in the LV is denoted as I (pressure filling wave), the propagation of the vortex wave is denoted as II. 